\title{
Cardiovascular risk assessment models: Have we found the perfect solution yet?
}

\author{
Aiden Abidov, MD, PhD, ${ }^{\mathrm{a}, \mathrm{b}}$ and Omar Chehab, MD, MSc ${ }^{\mathrm{a}}$ \\ a Division of Cardiology/Department of Internal Medicine, Wayne State University, Detroit, MI \\ b Cardiology Section/Department of Internal Medicine, John D. Dingell VA Medical Center, \\ Detroit, MI
}

Received Jan 27, 2019; accepted Jan 28, 2019

doi: $10.1007 / \mathrm{s} 12350-019-01642-\mathrm{x}$

\section{See related artical, pp. 2365-2374}

\section{INTRODUCTION}

Despite technical and pharmaceutical advancements and achievements in the last two decades and some decrease in overall mortality rate from cardiovascular disease (CVD), it remains the leading cause of death and economic burden. ${ }^{1}$

Among all other reasons, this problem may be attributed to the inability to control risk factors leading to $\mathrm{CVD}^{2,3}$ According to the INTERHEART study, a case-control study that enrolled over 29,900 subjects, there are nine significant modifiable risk factors contributing to cardiovascular disease: smoking, low fruit and vegetable intake, exercise, hypertension, diabetes, abdominal obesity, psychosocial factors, and lipid levels $(P<0.0001){ }^{4}$

In addition, more than $80 \%$ of patients with established CVD have a history of at least one of these major modifiable risk factors: smoking, hypertension, and dyslipidemia. ${ }^{5}$ Major non-modifiable and modifiable risk factors of CVD are summarized in Figure 1.

A clear understanding a patient's pre-test or pretreatment probability of a significant cardiovascular

\footnotetext{
Reprint requests: Aiden Abidov, MD, PhD, Cardiology Section/ Department of Internal Medicine, John D. Dingell VA Medical Center, 4646 John R., Detroit MI 48201, USA; aiden.abidov@wayne.edu

J Nucl Cardiol 2020;27:2375-85.

$1071-3581 / \$ 34.00$

Copyright (C) 2019 American Society of Nuclear Cardiology.
}

disease or adverse cardiac outcome (such as death or $\mathrm{MI}$ ), is an important part of the daily clinical decisionmaking process we encounter in our practice. Of course, we want to have prognostic assessment tools we can utilize with maximal available precision, especially when we estimate future cardiovascular risk. However, extensive evidence gained from the research based on large patient population databases in the late 20th century, demonstrated incremental value of multivariable risk assessment models as compared to a prognostic assessment of cardiovascular risk using just a few demographic variables. Furthermore, in the last decade, the concept of treating patients based on separated individual risk factors has changed to a management concept based on the patients' overall/global cardiovascular risk. ${ }^{5}$ With the advent of multivariable prognostic assessment tools, individual CVD risk factors have shown to follow a synergistic and multiplicative effect on global cardiovascular risk rather than an additive effect. ${ }^{6,7}$ However, novel risk factors and prognostic markers are emerging and are currently being implemented for optimal risk stratification, such as coronary artery calcium score, blood and/or urine biomarkers, and ethnicity. ${ }^{8-12}$ This has led to the derivation and validation of more than 50 cardiovascular risk predictive models. ${ }^{13,14}$ We summarized some of these models in Table 1. The importance of risk stratification allows clinicians to manage patients' risk factors according to their global cardiovascular risk by applying cost-effective, preventive, and medical measures while simultaneously considering the cost-to-benefit risk ratio. Since CVD is a consequence of multiple risk factors, the optimal risk assessment tools are those that provide physicians with a total cardiovascular risk, allowing clinicians to tailor their management accordingly. It is extremely important to utilize appropriate predictive models for specific prognostic goals; thus, a prognostic model created for prediction of obstructive CAD should 

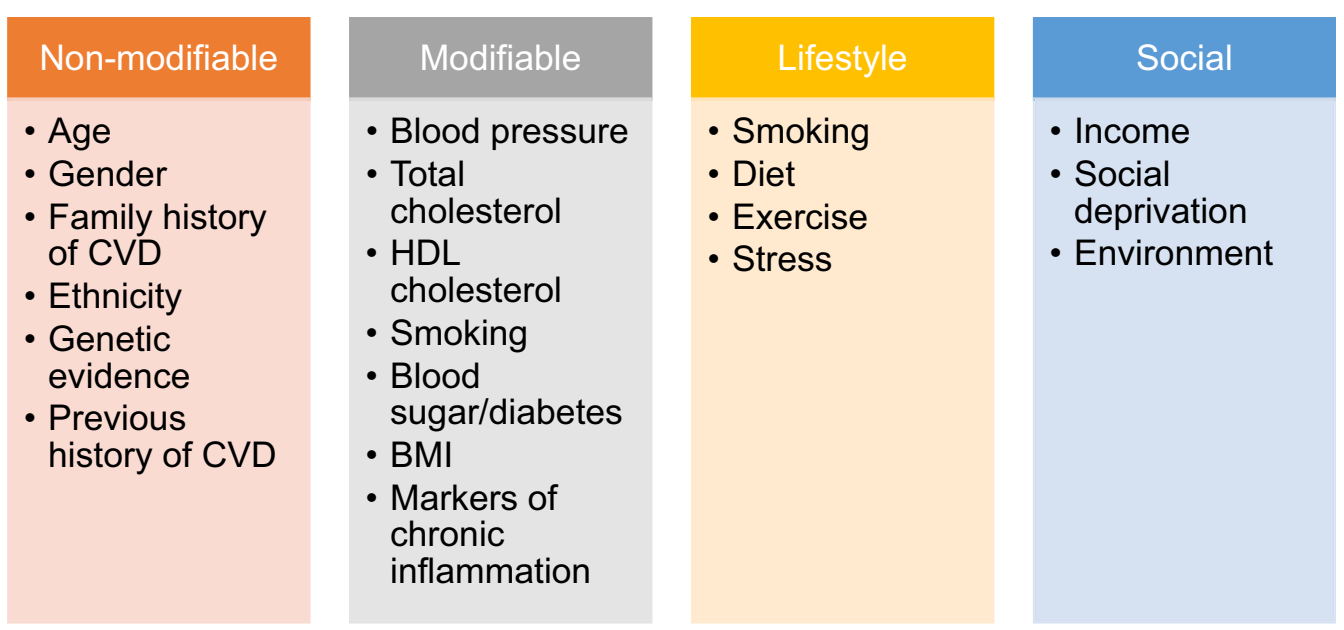

Figure 1. Demographic, historical, and clinical factors associated with increased risk of developing CVD.

not be used to predict future outcome (especially mortality) and vice versa. ${ }^{15}$

As shown in the Table 1, the majority of traditional CVD risk assessment tools are based on a consideration of demographic and historical data, while newer predictive models implement concept of multivariable analysis with a more complex selection process of the most powerful predictive variables included in the model.

A more sophisticated predictive risk analysis is frequently based on a stepwise forward logistic regression model, with the initial model including demographic/non-modifiable variables (Figure 1), and adding historical, hemodynamic, and imaging data on each subsequent step. ${ }^{16,17}$ Calculation of added predictive power of the model (expressed as a total Chisquare) in prior imaging studies has clearly shown a significant incremental predictive value of the model after counting in imaging data (such as coronary calcium and cardiac CTA results) on top of all other "traditional" risk factors of CVD. ${ }^{18}$

In this regard, many previously published papers from well-known research centers utilized risk assessment models on imaging data from the studies read by experts in the field. A difference in reading/interpretation of the imaging studies by imagers with a different level of expertise may lead to a lack of standardization in imaging data acquisition, and to a potential difference in the accuracy of total CVD risk assessment, while taking in consideration imaging data obtained in different medical centers.

In this issue of the Journal, Martineau et al, described a newly developed and validated cardiovascular risk assessment tool (CRAX) utilizing both clinical and automatically analyzed single photon emission computed tomography (SPECT) myocardial perfusion imaging (MPI) variables for predicting acute myocardial infarction (AMI) or death in patients at risk for coronary artery disease (CAD). ${ }^{19}$ The authors retrospectively created a validation set and analyzed the effect of adding the myocardial perfusion variable as an additional imaging variable to the clinical variables and looked at the net reclassification risk effect. The following were their respective conclusions ${ }^{19}$ :

A. Overall, the two predictive models using the CRAX for AMI and death outcomes proved to be more accurate in capturing events when using the combination of clinical variables and automated MPI results compared to either one variable alone.

B. When applying the Net Reclassification Improvement (NRI) analysis, there was an improvement in terms of risk stratification of patients who developed or had not developed events into lower or higher risk in both models of the CRAX after combining the clinical and automated MPI variables.

C. The common CRAX predictors for both AMI and death included age, number of recurrent hospitalizations in three years prior to MPI, and left ventricular ejection fraction. Additional predictors for AMI were transient ischemic dilation and ischemic total perfusion deficit while other predictors for death were utilization of pharmacological stress test and global stress total perfusion deficit.

Strengths of the article include the authors' selection of the combination of both clinical and automatically calculated imaging parameters for predicting AMI and death. ${ }^{19}$ Previous studies have 


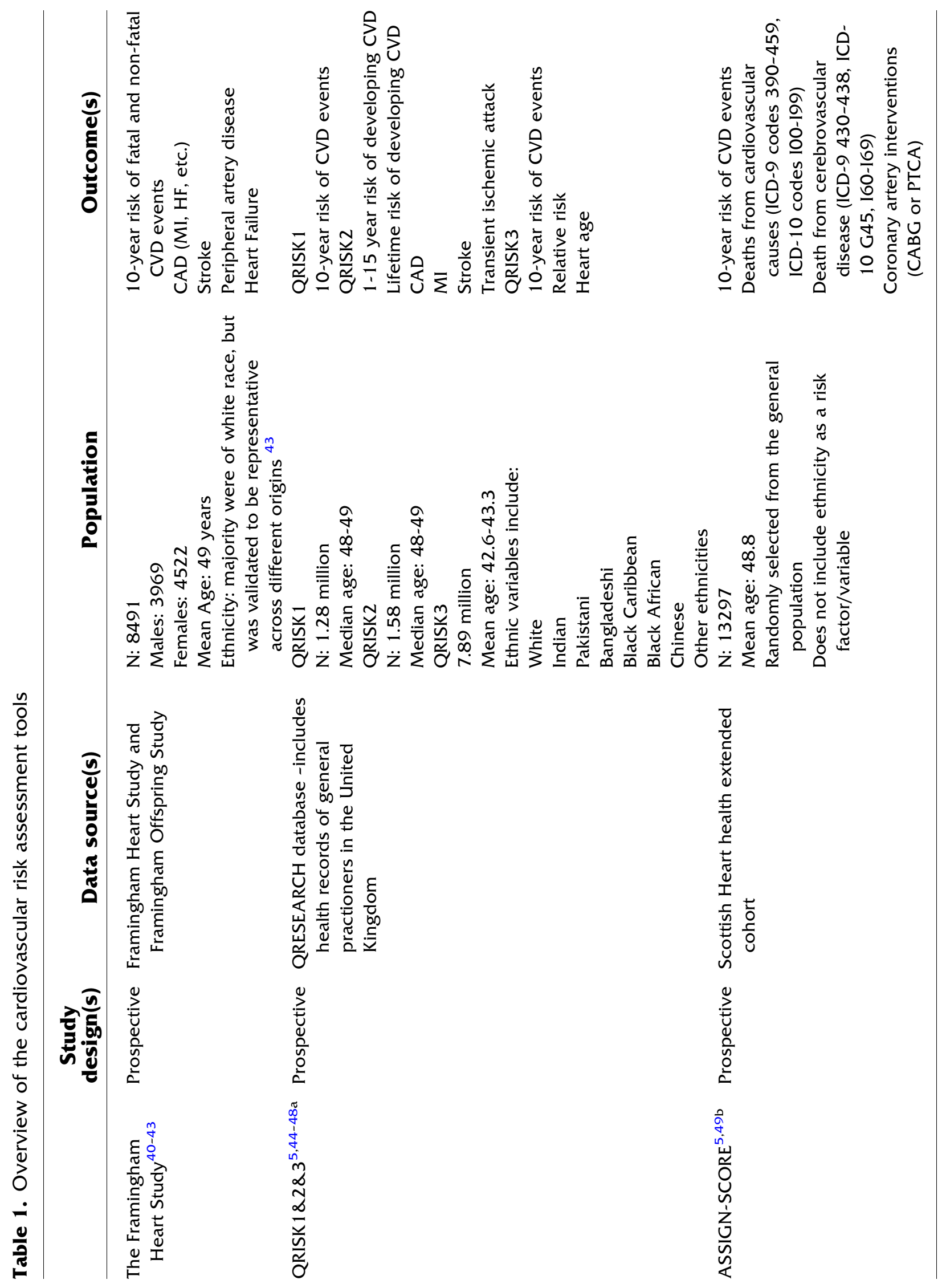




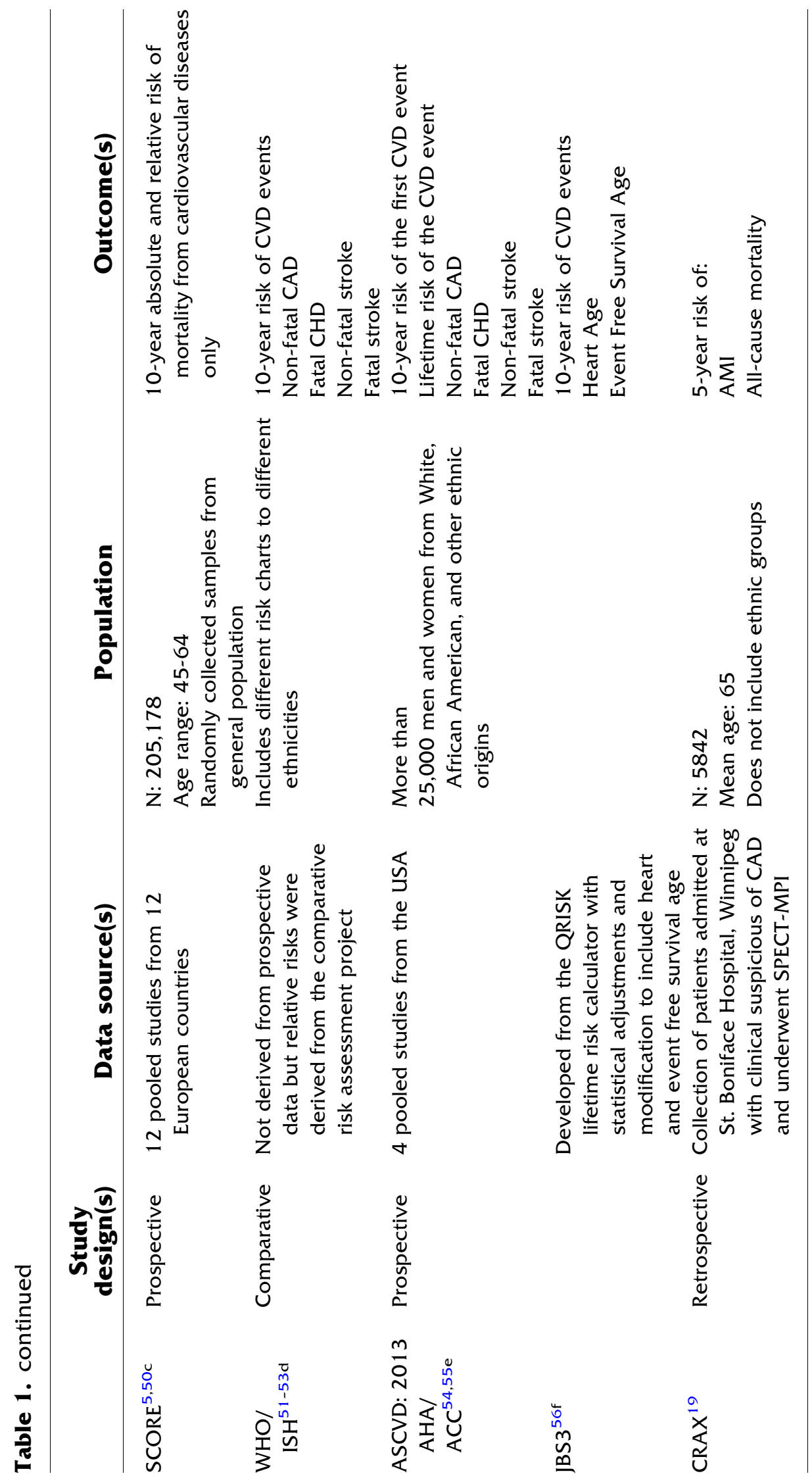



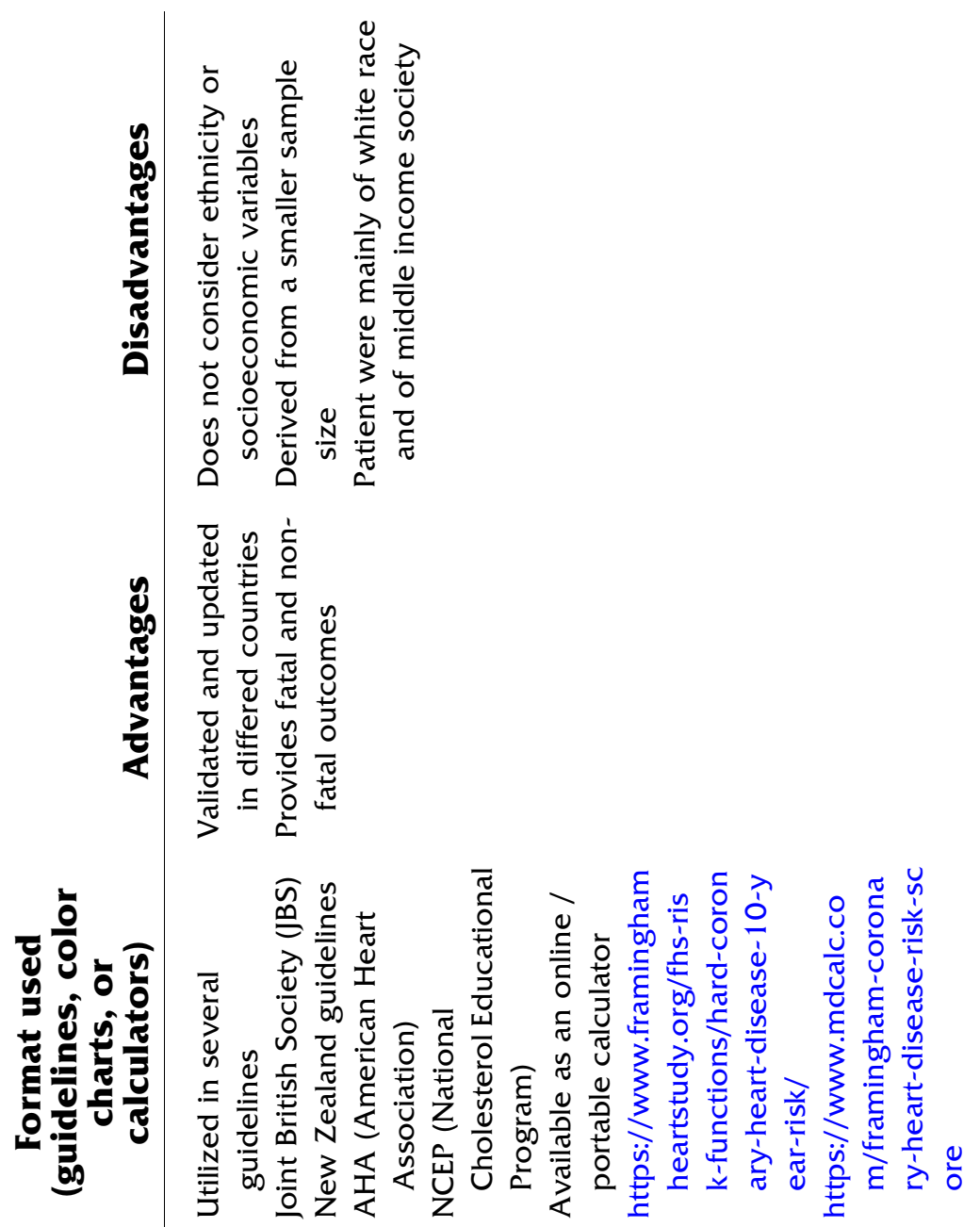

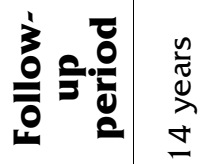




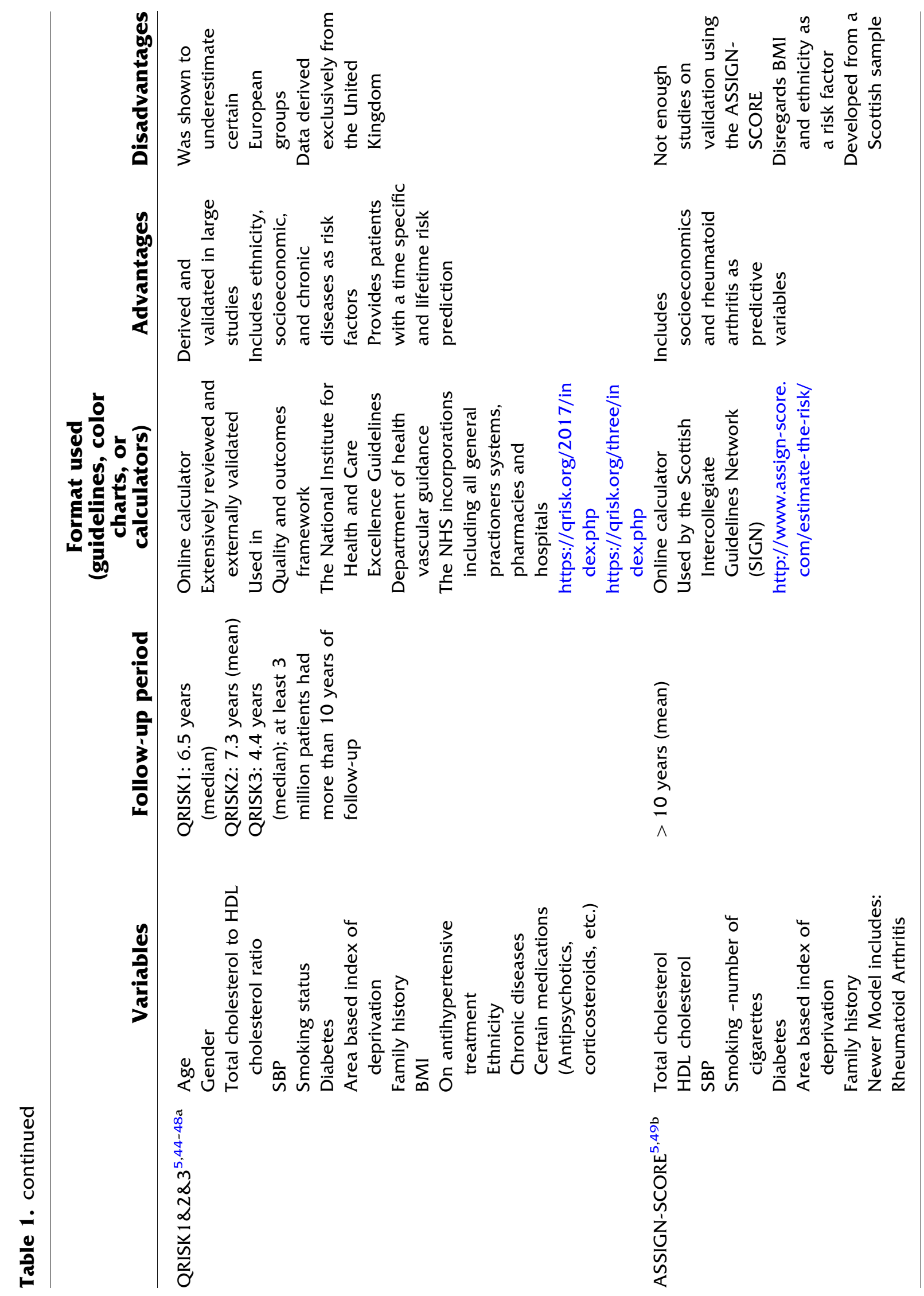



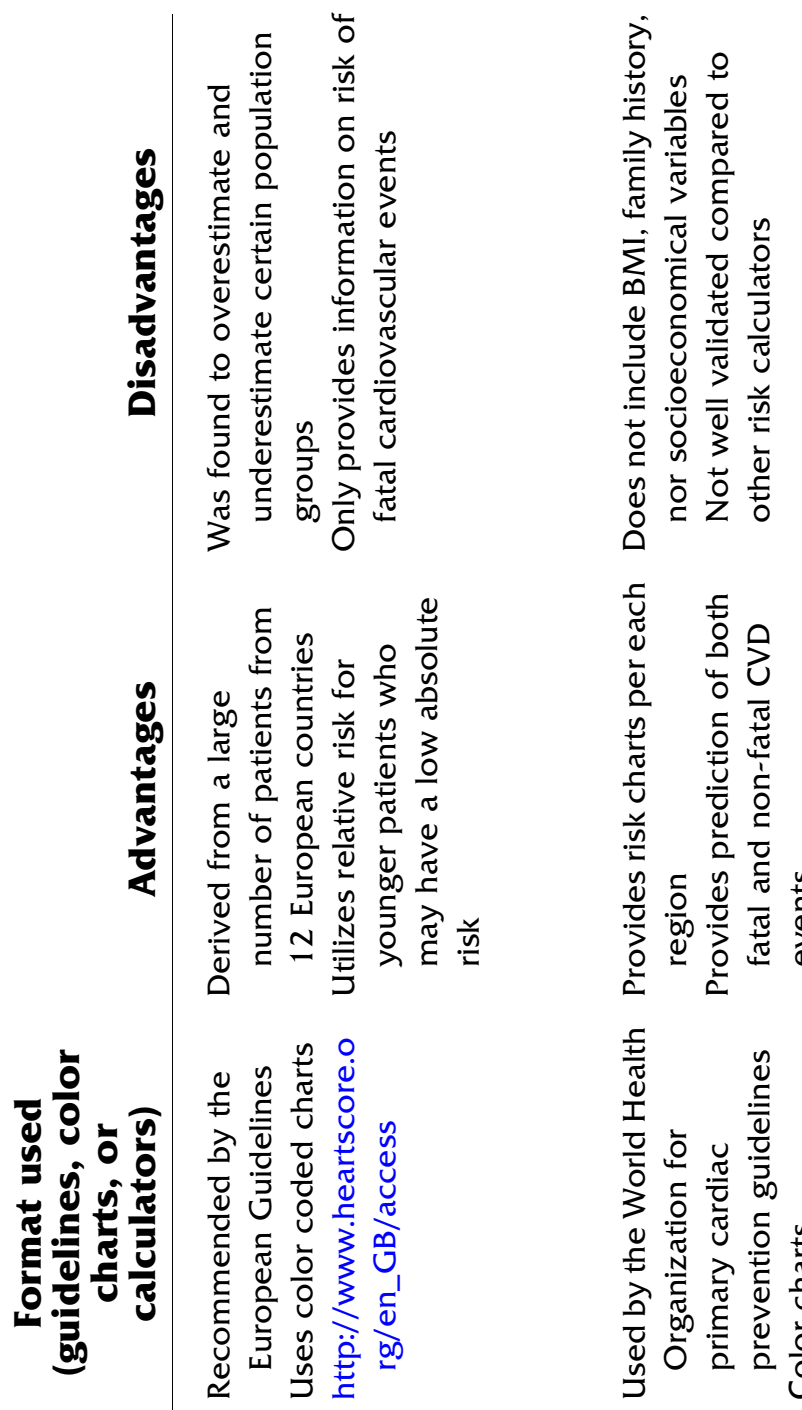

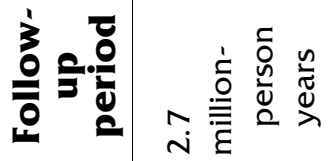
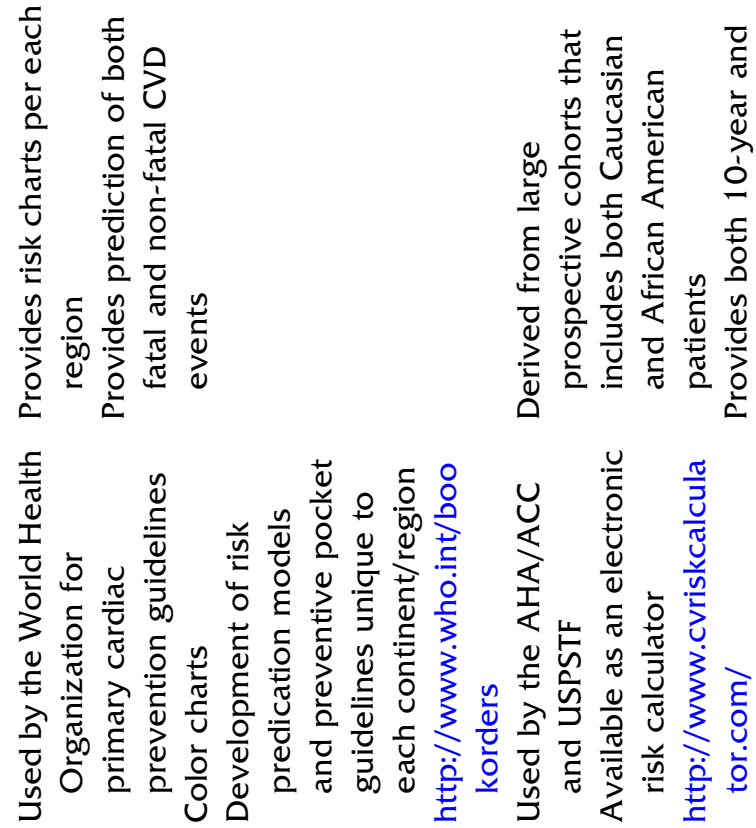

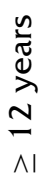
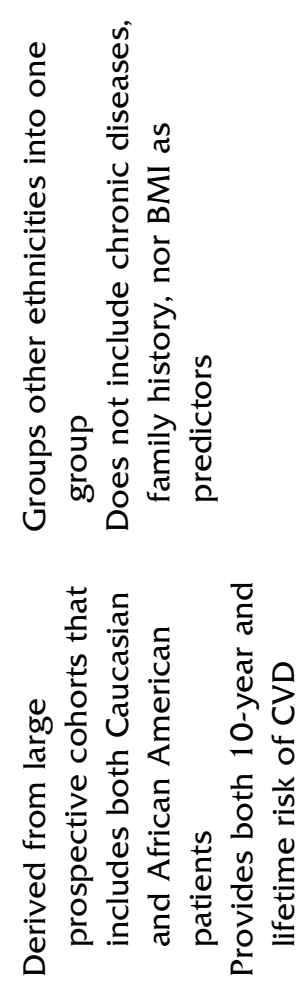
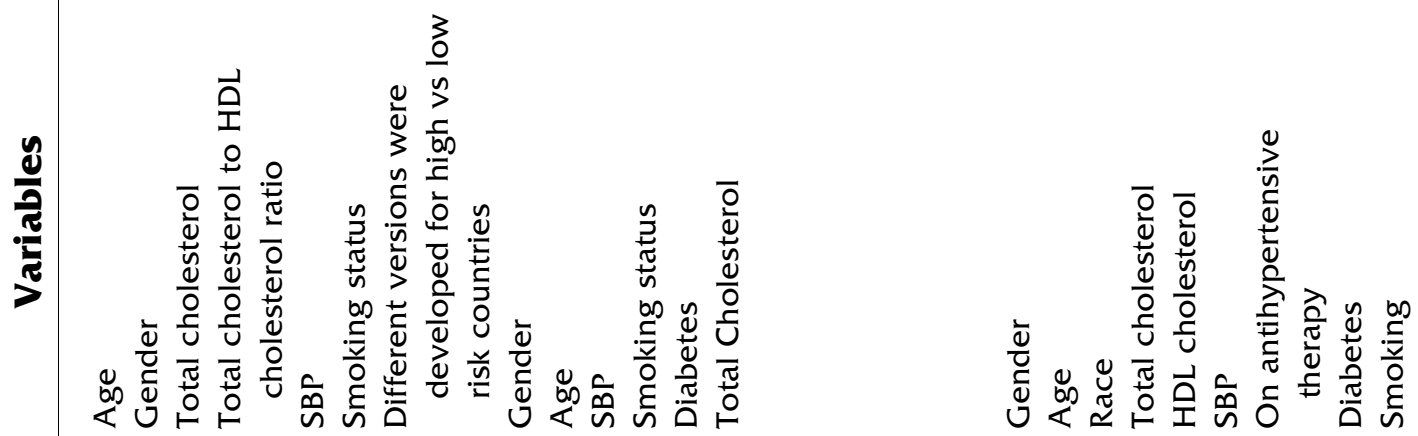

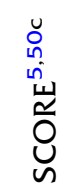

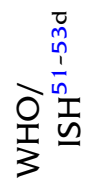




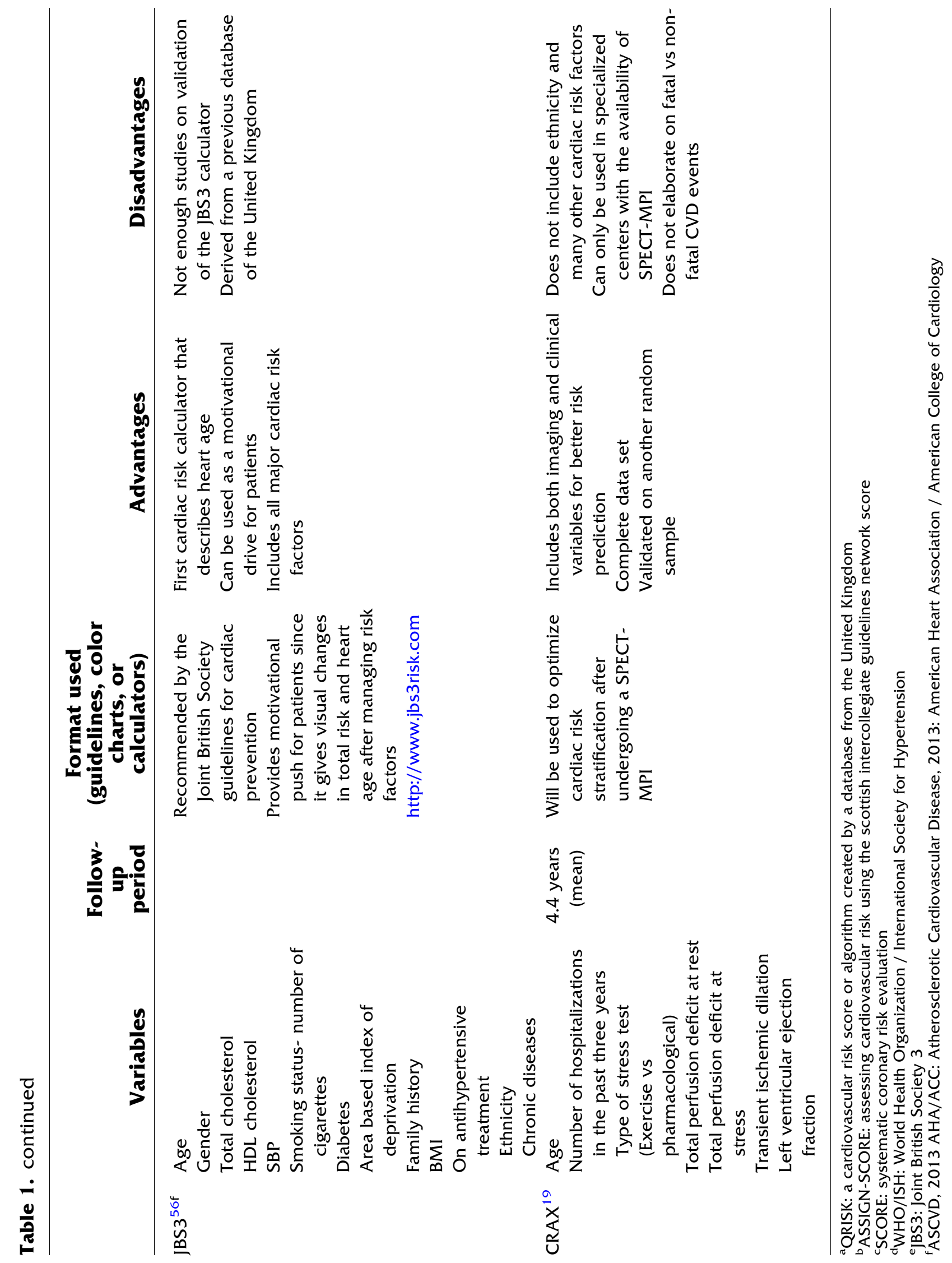


highlighted that the combination of imaging and clinical variables showed better results in terms of predicting acute cardiovascular events. ${ }^{20-22}$ A similar concept was developed using the coronary artery calcium (CAC) score in improving risk stratification when combined with a cardiac risk calculator/predictor. ${ }^{23}$ A landmark study by Detrano et al, found that when adding the clinical result of the CAC score to traditional cardiac risk factors, there was a clinical significant improvement in predicting adverse coronary events. ${ }^{24}$ The importance of combining a predictor marker such as the CAC score to traditional risk factors was highlighted in the multiethnic study of atherosclerosis (MESA) by Lakoski et al, that found that in asymptomatic women who were labeled as having a low Framingham risk score (FRS) with a detectable CAC were found to have a higher risk of developing coronary heart disease (CHD) compared to women with no detectable CAC and low FRS (hazard ratio [HR], 6.5; 95\% CI 2.6-16.4; HR, 5.2; 95\% CI 2.5$10)^{25}$

Another strength that was highlighted by the authors is the use of the net reclassification index (NRI) methodology. ${ }^{19}$ The NRI was first presented in 2008 by Pencina et al; its main purpose was to show that by adding new clinical variables to an existing prediction model, it will provide better risk stratification. ${ }^{26,27}$ Recently, NRI is frequently utilized in cardiovascular research, especially with the use of new biomarkers. ${ }^{26,28}$ In this study, the greatest re-stratification effect was on death, with the total NRI of $14.5 \%(P<0.001)$, emphasizing that the CRAX tool provided better prognostic reclassification when combining both clinical and imaging variables. A less impressive result was observed in patients with AMI, where the total NRI was $7.5 \%$. $(P$ $=0.046)$ The CRAX tool was also able to provide improved risk recertification in patients with no events, reclassifying these patients into the lower risk categories.

However, similar to other previously described CV risk assessment tools, the CRAX tool is not perfect. ${ }^{19}$ For example, CRAX did not include hemodynamic variables or ethnicity as a risk factor for developing CAD. The association of ethnicity as a novel risk factor for cardiovascular disease has been well-established in the literature. ${ }^{29-31}$ A study by Meadows et al, compared major cardiovascular event rates among patients with atherothrombotic disease in 46,602 individuals from 44 countries from the REACH Registry and found that the major cardiovascular event rates among patients with atherothrombotic disease were $4.5 \%$ of whites vs $6.1 \%$ of blacks and were lowest for all three Asian groups $(1.8-2.2 \%) .^{32}$ Since the 2013 ACC/AHA risk calculator lacks validation on a population other than whites and African Americans, a study by Rana et al, evaluated the applicability of the 2013 ACC/AHA risk calculator on more than 300,000 non-diabetic patients of diverse ethnicity (Blacks Asian/Pacific Islanders, and Hispanics) and with no prior history of CVD or use of statins; they found that the 2013 ACC/AHA risk calculator overestimated the risk of developing CVD and that was consistent among all ethnic groups. ${ }^{33-35}$ The same finding was consistent in two other studies that evaluated the validity of the ASCD risk calculator on patients from the MESA (Multi-Ethnic Study of Atherosclerosis) and the REGARDS (Reasons for Geographic and Racial Differences in Stroke) sample. ${ }^{36,37}$ Moreover, this has led to a recent update of the ASCVD risk calculator which brought an improvement to the validity of ASCVD risk calculator. ${ }^{38}$

In summary, the study by Martineau et al, presents a new perspective approach for cardiac risk stratification. By combining both clinical and automatically calculated MPI variables, the CRAX tool is able to achieve a more powerful CVD risk prediction score compared to either variable alone. It is very important to consider a possible variability in the MPI accuracy among different medical centers. Use of the CRAX cardiovascular risk assessment tool with the automated calculation of perfusion defect eliminates challenge of variable access to a good quality MPI and a lack of standardization in the MPI interpretation (and any other imaging study when used for risk stratification). In addition, the role of professional societies guiding the methodology, acquisition, and interpretation of the cardiac imaging studies in a standardized fashion as well as use of machine learning/ artificial intelligence becomes an important factor in the overall improvement of the CVD outcomes prediction in modern clinical practice. ${ }^{39}$

\section{Disclosure}

The authors have no relevant or material financial interests that relate to the research described in this paper.

\section{References}

1. Benjamin EJ, et al. Heart disease and stroke statistics-2018 update: A report From the American Heart Association. Circulation 2018;137:e67-492.

2. Frohlich ED, Quinlan PJ. Coronary heart disease risk factors: Public impact of initial and later-announced risks. Ochsner J 2014;14:532-7.

3. Willadsen TG, et al. The role of diseases, risk factors and symptoms in the definition of multimorbidity: A systematic review. Scand J Prim Health Care 2016;34:112-21.

4. Yusuf S, et al. Effect of potentially modifiable risk factors associated with myocardial infarction in 52 countries (the 
INTERHEART study): Case-control study. Lancet 2004;364:93752 .

5. Hobbs FD, et al. Barriers to cardiovascular disease risk scoring and primary prevention in Europe. QJM 2010;103:727-39.

6. Graham I, et al. European guidelines on cardiovascular disease prevention in clinical practice: Full text. Fourth Joint Task Force of the European Society of Cardiology and other societies on cardiovascular disease prevention in clinical practice (constituted by representatives of nine societies and by invited experts). Eur J Cardiovasc Prev Rehabil 2007;14:S1-113.

7. British Cardiac S, et al. JBS 2: Joint British Societies' guidelines on prevention of cardiovascular disease in clinical practice. Heart 2005;91:v1-52.

8. Chaturvedi N. Ethnic differences in cardiovascular disease. Heart 2003;89:681-6.

9. Kurian AK, Cardarelli KM. Racial and ethnic differences in cardiovascular disease risk factors: A systematic review. Ethn Dis 2007; 17:143-52.

10. Gilstrap LG, Wang TJ. Biomarkers and cardiovascular risk assessment for primary prevention: An update. Clin Chem 2012;58:72-82.

11. Vasan RS. Biomarkers of cardiovascular disease: Molecular basis and practical considerations. Circulation 2006;113:2335-62.

12. Jin J. Risk assessment for cardiovascular disease with nontraditional risk factors. JAMA 2018;320:316.

13. Bonner $\mathrm{C}$, et al. Clinical validity, understandability, and actionability of online cardiovascular disease risk calculators: Systematic review. J Med Internet Res 2018;20:e29.

14. Beswick AD, et al. A systematic review of risk scoring methods and clinical decision aids used in the primary prevention of coronary heart disease (supplement). London: Royal College of General Practitioners; 2008.

15. Naghavi M, et al. From vulnerable plaque to vulnerable patient: Part III: Executive summary of the Screening for Heart Attack Prevention and Education (SHAPE) Task Force report. Am J Cardiol 2006;98:2H-15H.

16. Shaw LJ, et al. A primer of biostatistic and economic methods for diagnostic and prognostic modeling in nuclear cardiology: Part II. J Nucl Cardiol 1997;4:52-60.

17. Shaw LJ, et al. A primer of biostatistic and economic methods for diagnostic and prognostic modeling in nuclear cardiology: Part I. J Nucl Cardiol 1996:3:538-45.

18. Abidov A, Raff GL. Value of coronary CTA in patients with known or suspected $\mathrm{CAD}$ and non-diagnostic initial myocardial perfusion testing: Current evidence and clinical considerations. J Nucl Cardiol 2010;17:1101-6.

19. Martineau P, Goertzen A, Leslie W. CRAX: A simple cardiovascular risk assessment tool to predict risk of acute myocardial infarction or death. J Nucl Cardiol 2018. https://doi.org/10.1007/ s12350-018-01556-0.

20. Betancur J, et al. Prognostic value of combined clinical and myocardial perfusion imaging data using machine learning. JACC Cardiovasc Imaging 2018;11:1000-9.

21. Koh AS, et al. Differential risk reclassification improvement by exercise testing and myocardial perfusion imaging in patients with suspected and known coronary artery disease. J Nucl Cardiol 2016;23:366-78

22. Shaw LJ, et al. Improved near-term coronary artery disease risk classification with gated stress myocardial perfusion SPECT. JACC Cardiovasc Imaging 2010;3:1139-48.

23. Osawa K, Nakanishi R, Budoff M. Coronary artery calcification. Glob Heart 2016;11:287-93.
24. Detrano R, et al. Coronary calcium as a predictor of coronary events in four racial or ethnic groups. $\mathrm{N}$ Engl $\mathrm{J}$ Med 2008;358:1336-45.

25. Lakoski SG, et al. Coronary artery calcium scores and risk for cardiovascular events in women classified as "low risk" based on Framingham risk score: The multi-ethnic study of atherosclerosis (MESA). Arch Intern Med 2007;167:2437-42.

26. Pencina MJ, D'Agostino RB Sr, Steyerberg EW. Extensions of net reclassification improvement calculations to measure usefulness of new biomarkers. Stat Med 2011;30:11-21.

27. Pencina MJ, et al. Evaluating the added predictive ability of a new marker: From area under the ROC curve to reclassification and beyond. Stat Med 2008;27:157-72 (discussion 207-12).

28. Hlatky MA, et al. Criteria for evaluation of novel markers of cardiovascular risk: A scientific statement from the American Heart Association. Circulation 2009;119:2408-16.

29. Karlamangla AS, et al. Socioeconomic and ethnic disparities in cardiovascular risk in the United States, 2001-2006. Ann Epidemiol 2010;20:617-28.

30. Hunt KJ, et al. All-cause and cardiovascular mortality among Mexican-American and non-Hispanic White older participants in the San Antonio Heart Study-evidence against the "Hispanic paradox". Am J Epidemiol 2003;158:1048-57.

31. Mensah GA, et al. State of disparities in cardiovascular health in the United States. Circulation 2005;111:1233-41.

32. Meadows TA, et al. Ethnic differences in cardiovascular risks and mortality in atherothrombotic disease: Insights from the Reduction of Atherothrombosis for Continued Health (REACH) registry. Mayo Clin Proc 2011;86:960-7.

33. Rana JS, et al. Accuracy of the atherosclerotic cardiovascular risk equation in a large contemporary, multiethnic population. J Am Coll Cardiol 2016;67:2118-30.

34. Jr. Smith SC, Grundy SM. ACC/AHA guideline recommends fixed-dose strategies instead of targeted goals to lower blood cholesterol. J Am Coll Cardiol 2014;64:601-12.

35. Cook NR, Ridker PM. Further insight into the cardiovascular risk calculator: The roles of statins, revascularizations, and underascertainment in the Women's Health Study. JAMA Intern Med 2014;174:1964-71.

36. Ridker PM, Cook NR. Statins: New American guidelines for prevention of cardiovascular disease. Lancet 2013;382:1762-5.

37. DeFilippis AP, et al. An analysis of calibration and discrimination among multiple cardiovascular risk scores in a modern multiethnic cohort. Ann Intern Med 2015;162:266-75.

38. Yadlowsky S, et al. Clinical implications of revised pooled cohort equations for estimating atherosclerotic cardiovascular disease risk. Ann Intern Med 2018;169:20-9.

39. Slomka PJ, et al. Cardiac imaging: Working towards fully-automated machine analysis and interpretation. Expert Rev Med Devices 2017;14:197-212.

40. D'Agostino RB Sr, et al. General cardiovascular risk profile for use in primary care: The Framingham Heart Study. Circulation 2008;117:743-53.

41. Kannel WB, et al. An investigation of coronary heart disease in families. The Framingham offspring study. Am J Epidemiol 1979;110:281-90

42. Dawber TR, Meadors GF, Moore FE Jr. Epidemiological approaches to heart disease: The Framingham Study. Am J Public Health Nations Health 1951;41:279-81.

43. D'Agostino RB Sr, et al. Validation of the Framingham coronary heart disease prediction scores: Results of a multiple ethnic groups investigation. JAMA 2001;286:180-7. 
44. Hippisley-Cox J, et al. Derivation and validation of QRISK, a new cardiovascular disease risk score for the United Kingdom: Prospective open cohort study. BMJ 2007;335:136.

45. Hippisley-Cox J, et al. Predicting cardiovascular risk in England and Wales: Prospective derivation and validation of QRISK2. BMJ 2008;336:1475-82.

46. Hippisley-Cox J, Coupland C, Brindle P. Development and validation of QRISK3 risk prediction algorithms to estimate future risk of cardiovascular disease: Prospective cohort study. BMJ 2017;357:j2099.

47. Minhas JS, Patel P, Gupta PK. Limitations of a cardiac risk (QRISK2) calculator in patients with high density lipoprotein (HDL). High Blood Press Cardiovasc Prev 2016;23:47-50.

48. Collins GS, Altman DG. An independent external validation and evaluation of QRISK cardiovascular risk prediction: A prospective open cohort study. BMJ 2009;339:b2584.

49. Woodward M, et al. Adding social deprivation and family history to cardiovascular risk assessment: The ASSIGN score from the Scottish Heart Health Extended Cohort (SHHEC). Heart 2007;93:172-6.

50. Conroy RM, et al. Estimation of ten-year risk of fatal cardiovascular disease in Europe: The SCORE project. Eur Heart J 2003;24:987-1003.
51. Organization WH. Prevention of cardiovascular disease guidelines for assessment and management of cardiovascular risk. Genenva: WHO; 2007.

52. Otgontuya D, et al. Assessment of total cardiovascular risk using WHO/ISH risk prediction charts in three low and middle income countries in Asia. BMC Public Health 2013;13:539.

53. Selvarajah $\mathrm{S}$, et al. Comparison of the Framingham Risk Score, SCORE and WHO/ISH cardiovascular risk prediction models in an Asian population. Int J Cardiol 2014;176:211-8.

54. Stone NJ, et al. 2013 ACC/AHA guideline on the treatment of blood cholesterol to reduce atherosclerotic cardiovascular risk in adults: A report of the American College of Cardiology/American Heart Association Task Force on Practice Guidelines. Circulation 2014;129:S1-45.

55. Goff DC Jr, et al. 2013 ACC/AHA guideline on the assessment of cardiovascular risk: a report of the American College of Cardiology/American Heart Association Task force on practice guidelines. Circulation 2014;129:S49-73.

56. Board J. Joint British Societies' consensus recommendations for the prevention of cardiovascular disease (JBS3). Heart 2014;100(Suppl 2):1-67.

Publisher's Note Springer Nature remains neutral with regard to jurisdictional claims in published maps and institutional affiliations. 